\title{
Análisis de la producción audiovisual y panorama musical en Extremadura a través de los canales de YouTube
}

\author{
David Delgado Martínez
}

Recibido: 10 de diciembre de 2015

Aceptado: 12 de febrero de 2016

http://dx.doi.org/10.5209/rev_CDMU.2016.v27.n1.51866

\section{Resumen}

Se lleva a cabo un estudio sobre el panorama musical extremeño y su producción audiovisual a través de la difusión por medio de la plataforma audiovisual YouTube. La finalidad de este estudio es tener un análisis de todos los productos audiovisuales, analizando cuáles son los procesos de difusión que los creativos llevan a cabo para dar a conocer sus obras tanto musicales como audiovisuales. Realizaremos un estudio cuantitativo del material audiovisual de las bandas extremeñas y además un estudio cualitativo sobre cuales son los procesos más idóneos para que los vídeos lleguen al mayor número de usuarios.

\section{Palabras clave}

Panorama musical; Cibercultura; Extremadura; YouTube; Trovadores digitales; Covers; Interacción creativa; Auto producción; Partners,

\section{Analysis of audiovisual production and music scene in Extremadura through YouTube channels}

\section{Abstract}

A study on Extremenian music and audiovisual production has been carried out through YouTube media. The purpose of this study is to analyze all audiovisual products and also assessing what broadcasting processes are conducted by creators to publicize their musical and audiovisual works. A quantitative and qualitative study of audiovisual materials of Extremadura bands was done to find out which processes are most suitable to increase the number of viewers for videos.

\section{Keywords}

Music scene, Cyberculture; Extremadura; YouTube; digital Troubadours; Covers; creative interaction; Auto production; Partners. 


\section{Introducción}

Las nuevas tecnologías y los nuevos medios de información y comunicación han planteado un nuevo escenario para la cultura. La cibercultura ha motivado que cada vez sean más los creativos que utilizan la red para dar a conocer sus producciones.

En el origen de las artes, la obra era emitida por un "emisor creativo" mediante un canal físico como podía ser un lienzo, un libro, un bloque de piedra, una película de celuloide u otro soporte que más tarde llegaba a un receptor que se encargaba de descifrar el mensaje y así recibir el contenido la obra. Podemos definir este proceso de comunicación como unidireccional. En cambio, en la actualidad, los nuevos medios de comunicación como YouTube (Antolin, 2012) (LISI, 2008) han creado una comunicación bidireccional en el que existe una comunicación directa entre el creativo y el espectador y viceversa. Produciéndose así, en muchas ocasiones un fedback muy interesante que hacen que las obras tomen una mayor dimensión tanto artística como cultural.

Ante esta realidad, nos encontramos con un importante problema, ya que no tenemos un control sobre toda la obra audiovisual-musical que se produce en nuestra región. Ante este problema podemos argumentar que lo realmente valioso creativamente acabará por destacar a lo largo del tiempo. Los historiadores del arte y la ciencia de la historia del arte, argumentan que una producción artística de un creativo determinado es buena cuando el filtro del tiempo y el estudio ponen de relieve aquellas obras de mejor calidad. Pero, ¿Cuántos magníficos artistas habrán muerto sin ser reconocidos? ¿Cuánta belleza se habrá perdido el ser humano? ¿Cuántas magníficas obras no han sido descubiertas por el público? ¿Cuánta producción audiovisual-musical producida en nuestra comunidad autónoma no está catalogada?

Quizás, el trabajo más importante de historiadores y documentalistas esté en hundirse en las profundidades de la cultura digital y sacar a flote lo que forma parte de nuestro patrimonio cultural. Un patrimonio cultural que en ocasiones es totalmente relegado al ostracismo y considerada cultura underground. Ya que se mueve por circuitos pequeños 
con un público minoritario. Fuera de las industrias culturales y de cualquier apoyo desde instituciones públicas. Pero, sin duda, forman parte de la identidad y la cultura contemporánea de nuestra región.

Lo que pretende este artículo es un análisis del panorama musical contemporáneo en Extremadura, a través de los canales de YouTube. Con la finalidad de entender como los nuevo músicos y creativos se las ingenian y se sirven de las herramientas que YouTube les proporciona para dar a conocer su material audiovisual.

El estudio está motivado, en primer lugar, porque no existe ningún tipo de estudio sobre el tema, y además con la finalidad de estudiar cuales son los procesos más importantes e idóneos que se han de llevar para difundir productos audiovisuales a través de la red.

\section{Los músicos digitales}

Las nuevas tecnologías han desarrollado un gran número de software de edición de sonido y vídeo que han permitido a los músicos llevar a cabo una producción musical de muy buena calidad en un pequeño estudio casero. Softwares como Cubase, Pro tools, o Logic de edición profesional de sonido junto a un interface de audio y una microfonía profesional junto a mucho trabajo y conocimientos en sonido en la actualidad permiten a un cantautor o banda llevar a cabo un trabajo discográfico de una calidad aceptable para mostrar en la red. Toda esta revolución tecnológica ha motivado que los creativos produzcan más contenidos y además mejorar la calidad, ya que cuentan con mucho tiempo para trabajar las canciones. Frente al trabajo bajo presión que se lleva en los estudios de grabación.

Ya lo decía Jorge Drexler en uno de sus versos de la canción "Guitarra y vos": "El cantautor y su computadora". Drexler pone de relieve la importancia del ordenador y de las nuevas tecnologías en la lírica contemporánea.

Además, la democratización de las nuevas tecnologías y el acceso a ellas ha permitido a 
muchos artistas confeccionar sus estudios caseros. Haciendo que el panorama musical sea más rico a nivel regional como estatal.

Los músicos, además de producir su material audiovisual se van a encargar de distribuir todo ese material auto producido a través de la red llegando a sus oyentes de un modo directo. Tenemos como ejemplo al cantautor madrileño Quique González que ha creado su propio sello discográfico bajo el que edita su producción discográfica.

Por ello, en la actualidad no solo encontramos creativos musicales si no que en muchas ocasiones se convierten en auténticos especialistas del marketing y del negocio discográfico. Motivado por la crisis que ha hecho que las discográficas desaparezcan e incluso encarguen al propio músico que produzca sus propios discos. Sea a causa de la industria o a la crisis, sin duda ha sido muy positivo tanto para los artistas como para la industria ya que hay una importante riqueza musical y una importante producción de disco anualmente.

Las redes sociales como Facebook, Twitter o Instragram son otras de las herramientas de las que se sirven los músicos. No solo con la subida de sus canciones y temas musicales sino que realizan una auténtica producción fotográfica (Негrera y Codina, 2015), comentarios, entradas, gif, animaciones, etc. Un buen ejemplo es la banda extremeña Los Ganglios (www.losganglios.es) quienes con mucha creatividad diseñan diferentes carteles que hacen que el público se interese más por sus contenido como por sus conciertos y actuaciones. Estos utilizan el humor y el sarcasmo tanto en sus composiciones como en la cartelería y vídeos musicales.

Esta banda cuenta con unas cifras muy importantes en su canal de YouTube (2.018.687 visualizaciones) que se ve reflejado en la creación de una comunidad de seguidores.

Definimos comunidad de seguidores como el grupo de fans que siguen a una banda determinada. Los fans o seguidores suelen ser muy activos en el comentario de vídeos, comparten los contenidos y además existe una interacción entre los diferentes fans. Creándose una auténtica "tribu digital" que siguen a un grupo determinado (Reguillo, 
Por otro lado, las nuevas bandas y cantautores generan un gran número de contenidos que también forma parte de su producción artística. Carteles, fotografías, vídeos, publicidad, gif., animaciones, vídeos colaborativos en los que sus fans forman parte del videoclip. Sin duda, enriquecen la producción artística de las bandas, convirtiéndose en creativos interdisciplinares que ponen en relación un gran número de habilidades creándose discursos artísticos interdisciplinares que enriquecen la comunicación con el espectador.

\section{Trovadores digitales}

Al desarrollar este trabajo hemos tenido que definir a los nuevos músicos como trovadores digitales. En la edad media la figura del trovador era muy reconocida socialmente e incluso en la historia de la literatura se tiene en muy buena consideración a estos autores. Ellos, fueron los grandes divulgadores de la cultura y la información de las sociedades de la edad media por los diferentes pueblos.

Como saben, los trovadores eran en la edad media músicos que recorrían las tierras cantando batallas, acontecimientos y noticias que se daban en las sociedades de la edad media. Haciendo una analogía con nuestra actualidad cultural serían los periodistas de nuestra actualidad. Se dedicaban a hacer una fotografía de la sociedad en sus composiciones. En forma de canciones, poemas, versos alejandrinos, etc. Recorrían las ciudades cuando los hombres habían ido a la batalla. Normalmente, cantaban para las mujeres de los soldados que habían partido a la batalla con el fin de informar sobre los acontecimientos acaecidos en el campo de batalla. También, cantaban sobre otros temas como historias de amor, chismes y coplas sobre la monarquía o la nobleza. Una de las grandes diferencias que existen con los juglares es que este último solo componía canciones, en cambio el trovador componía e interpretaba sus composiciones. En muchas ocasiones con una actitud errante y bohemia. Eran hombres desarraigados, su instrumento y voz eran su equipaje, y a pie o sobre una mula recorrían los campos y ciudades informando y entreteniendo a los aldeanos. 
En la actualidad, autores como Bob Dylan, Leonard Cohen, Jonhny Cash, Joaquín Sabina, Joan Manuel Serrat, Devendra banhart, Carla Morrison, Jorge Drexler, Pablo Guerrero o Luís Pastor como muestra de nuestra cultura popular extremeña. Son los nuevos trovadores postmodernos, que con su guitarra bajo el brazo y en muchas ocasiones armados con las nuevas tecnologías recorren las ciudades y pueblos con su voz y guitarra contando batallas, historias de amor y desamor, sobre política, chismes sobre reyes y nobles. En realidad, muy poco ha cambiado desde la edad media. Pero las nuevas tecnologías han abierto más el canal de comunicación entre los músicos y el público. El cantautor postmoderno tiene herramientas que le permite una comunicación global. Desde su pequeño estudio puede lanzar sus composiciones llegando a miles de internautas que reciben sus composiciones e interactúan con ellas.

\section{Cultura Global}

Muchos de los contenidos que los creativos generan son compartidos por los espectadores y convirtiéndose en vídeos virales que recorren redes sociales, blogs, chats y otras aplicaciones haciendo que los contenidos se conviertan en contenidos populares.

Por ello, nos encontramos en una cultura global, en el que un cantautor no solo se vincula a su lugar geográfico de procedencia. Si no que su obra rompe fronteras, pudiendo llegar sus contenidos a cualquier lugar del mundo. Hemos de hablar de la Cibercultura, como la nueva cultura imperante en las sociedades de la información y la comunicación. No podemos hablar de cultura regionalista y cerrada sino que todas las tendencias artísticas ya sean pintura, música, escultura, vídeo, cine, etc. están globalizadas. Los estilos y tendencias se contaminan y se nutren de otros, creándose artistas tan interesantes como Devendra Banhart. Cantautor de origen chileno pero afincado en los Estados unidos de américa desde muy joven. Banhart se dedica a la canción y al diseño gráfico. Siendo uno de los artistas más interesantes del panorama norteamericano. Devendra, según argumenta en una de sus entrevista dice que se basa e inspira en cantautores latinos como Atahualpa Yupanki, Caetano Beloso, etc. Y además 
que en sus canciones trata de hacer del texto cantado una obra visual. Es decir, cada verso es creado a partir de una imagen mental que se contiene en una palabra. Es aquí el ejemplo de que los nuevos artistas rompen las disciplinas artísticas y las fusionan enriqueciéndose así el lenguaje y la comunicación. Las obras musicales son poliédricas, encontramos vídeos, fotografía, diseños, páginas web, arte de los discos, etc. Haciendo mucho más difícil catalogar toda la producción de un artista.

Por ello, la música y la cultura actual, es global. Sería un auténtico error entender la música de nuestra comunidad sin estar abierto a las influencias de otros países.

Sí es verdad, que existen unas formas de hacer que nos identifican como cultura. Como puede ser la lengua, las formas de componer, los timbres de los instrumentos tradicionales como la guitarra de flamenco, el cajón de flamenco, los giros vocales que recuerdan a la música tradicional y popular de Extremadura y Andalucía. E incluso, la utilización de versos de cantos populares, bandas como Extremoduro ha utilizado cantos populares para introducir en sus discos.

Quizás, lo que más nos identifique como pueblo sean los núcleos de las composiciones musicales, mientras que muchos arreglos, ritmos, efectos, sintetizadores, estéticas, etc. Son de influencia externa. Principalmente de la música anglosajona. Por ello, no podemos decir que exista una música con carácter extremeño sino que es una contaminación de muchos estilos que forman parte de la cultura global o Cibercultura.

\section{Cibercultura}

La Cibercultura es el gran cambio que se ha dado en la actualidad en las artes. Este nuevo panorama cultural ha motivado que los artistas cada vez tengan que ser más hábiles y deben servirse de otras artes para que su discurso sea interesante. Por lo que hemos de hablar del artista multidisciplinar o el "neo hombre renacentista". La historia del arte, dio durante el siglo XV en el periodo artístico del renacimiento la figura del artista universal también llamado hombre del renacimiento. Este hombre se caracterizaba por ser interdisciplinar y servirse de muchas artes para llevar sus 
producciones a buen puerto. Auténticos genios como Leonardo da Vinci o Miguel Ángel son un buen ejemplo de hombres del renacimiento. Sin duda alguna Leonardo da Vinci, es el buen ejemplo de artista renacentista. Por todos es conocido sus conocimientos sobre física, pintura, dibujo, anatomía, e incluso creador de inventos que fueron inspiración para muchos de los inventos que se han llevado posteriormente.

En la actualidad, a causa de la gran producción artística y la existencia de diferentes medios de comunicación de los que cuentan los artistas se demandan un gran número de artistas interdisciplinares. Es decir, un músico si quiere llegar más a la gente no solo debe seguir un arte como puede ser la música, si no que ha de servirse de otras técnicas como el vídeo, la fotografía, el videoclip, la performance, etc. Para que los contenidos lleguen de un modo más atractivo a su público.

Las nuevas tecnologías han creado nuevos lenguajes artísticos haciendo que las artes se fusionen, se entremezclen. La fotografía se mezcla con el vídeo, el teatro con el cine, el cine con la música. Es decir, existe una mezcla de lenguajes artísticos que enriquecen la obra. Pero además, requiere de los artistas una mayor formación y creatividad. En la actualidad, un músico no solo ha de entrar en un estudio de grabación de sonido y ahí termina su trabajo. Si no que ha de armar un discurso conceptual que de sentido completo a la obra.

Por ejemplo, un cantautor, ha de componer las canciones, la música, la letra pero más tarde deberá crear el arte del disco, cartelería, la publicidad, diseño de banners, fotografías de promoción y todos los contenidos audiovisuales como pueden ser videoclips, actuaciones en directo, conciertos acústicos, reportajes y documentales sobre giras y actuaciones. Esto sin duda pone en funcionamiento una gran maquinaria creativa y de producción, en el que muchas ocasiones es el propio artista el que ha de elaborarla o en otras ocasiones se necesita de otros creativos como diseñadores, editores de vídeo, realizadores, ilustradores, fotógrafos, etc.

En la actualidad, editar un disco y que no exista un vídeo de al menos una canción es algo extraño. Si no es así, es un disco que no va a tener visibilidad en el ciberespacio. Porque 
nuestra cultura actualmente, es totalmente audiovisual. El arte, ya no es estático. El cuadro que cuelga en el museo ha de moverse para atraer, para ser atractivo para el gran público de las nuevas generaciones. Vivimos en la era artística de la imagen en movimiento, de la interacción, de la intervención, de la performance e implicación del espectador en la obra.

\section{Objetivos y metodología}

El objetivo principal de este estudio es demostrar como un gran número de bandas extremeñas se sirven de las redes sociales para dar a conocer su producción audiovisual. Realizaremos un estudio cuantitativo de todas las cifras de cada una de las bandas que tienen canal en YouTube. Por un lado, vamos a tomar notas de las cifras de cada una de las bandas durante el mes de octubre y noviembre de 2015 y elaboraremos un ranking de las bandas extremeñas de mayor a menor cifra. Y así, entender cuales son las causas las que motivan que una banda esté más arriba o más abajo en dicho ranking.

Por otro lado, la finalidad de este estudio es hacer un inventario de las bandas más destacadas de música popular en Extremadura que actualmente están en activo con disco editado y con contenidos audiovisuales en los canales de YouTube.

Por ello, este artículo tiene como objetivo principal un análisis cuantitativo de las cifras sobre contenidos audiovisuales en YouTube. Pero también, quiere ser un resumen de la escena musical popular de los últimos años en nuestra región.

El método empleado se ha implementado por medio de fases:

\section{Primera fase}

En primer lugar, con el fin de acotar el número de bandas a estudiar y dar un rigor científico a la investigación, hemos desarrollado un criterio que las bandas y autores han de tener рага formar parte del estudio. Este criterio consiste en que las bandas o autores deben de tener al menos un disco editado con registro en la propiedad intelectual o depósito legal durante los dos últimos años (2014-2015). Además, deben de contar con 
un canal de YouTube con al menos un vídeo profesional subido a la plataforma. Se considera vídeo profesional, aquellos que tienen una calidad técnica y artística, no admitiéndose grabaciones caseras, de conciertos o actuaciones tomados con un dispositivo móvil o cámaras de baja calidad sin ningún tipo de postproducción audiovisual.

Una vez, realizado las búsquedas por la red y asegurándonos de que se cumplían ese criterio, realizamos un listado con las bandas extremeñas que contaban con dichos requisitos. Esta primera fase, nos permite acotar nuestro campo de estudio hacia aquellas bandas que tienen una actividad profesional o al menos semi profesional.

El listado de bandas que hemos desarrollado es el siguiente, ordenado por orden alfabético:

Acetre, Alé Canalla, Bebe, Bucéfalo, Cajón de Sastre, Cira \& Ulises, Chloe Bird, Da, Divan du don, Duende Josele, Extremoduro, Los Ganglios, Gecko Turner, Hemingway, Inlavables, Javier Alcántara, Kaxta, Los niños de los ojos rojos, Luis Pastor, Marcos Bayón, Mili Vizcaíno, Olivenza, Omnia Transist, Pablo Guerrero, Robe, Rubén Rubio, Rui Díaz y la banda imposible, Sínkope, Subterráneos y Supertennis.

\section{Segunda fase}

En la segunda fase vamos a elaborar un análisis del número de suscriptores con los que cuenta el canal de cada una de las bandas y además el número de vídeos que están subidos al canal y el número total de visualizaciones. Mediante una tabla nos permitirá analizar los diferentes datos de cada una de las bandas, y así crear un ranking u orden de mayor a menor visualizaciones. Pudiendo comparar el número de contenidos en el canal con el número de visualizaciones.

\section{Tercera fase}

En esta fase vamos a analizar el nivel de interacción entre las bandas y autores con los usuarios, analizando la frecuencia temporal en la que suben contenidos al canal. Los analizaremos del siguiente modo: "vídeos subidos hace dos años", "vídeos subidos hace 
un año", "vídeos subidos hace 6 meses", "vídeos subidos hace menos de seis meses".

También analizaremos si existe interacción entre el público con los contenidos subidos al canal. Llevando la contabilidad de los mensajes escritos en sus vídeos y la interacción entre autores y público.

\section{Cuarta fase}

A pesar de que los canales de YouTube son un buen escaparate para distribuir los contenidos para los creativos hacia sus usuarios, el "medio de comunicación" YouTube es una herramienta compleja para crear unas cifras altas de visualizaciones. Por lo que las grandes cifras de visualizaciones son solo conseguidas por una serie de empresas de la industria audiovisual que mediante el pago de publicidad y colocación de banners en determinados lugares de las páginas hacen que los contenidos audiovisuales lleguen al gran público.

Trataremos de desmontar la idea de cómo YouTube es un medio que llega al gran público con solo colgar contenidos en sus canales. Se analizará cuáles son los procesos más idóneos para que los contenidos de un canal tengan un importante tráfico de usuarios y así suban las visualizaciones de los vídeos del canal.

Realizaremos el estudio sobre aquellas bandas que al menos tienen un disco editado y además cuentan con un canal de YouTube para dar a conocer sus trabajos. Analizaremos cuales son los intervalos temporales de actualización de los contenidos. Tratando de demostrar que el número de visitas a los canales y a los vídeos está directamente relacionado con la periodicidad de publicación de contenidos y además de una distribución inteligente y creativa a través de redes sociales como Facebook, Twitter, Instagram, entre otras. $\mathrm{Y}$ como aquellos, grupos que mejor tienen planteado su estrategia en la red tiene mejores resultados cuantitativos. 


\section{Resultados}

Un resumen de resultados es el señalado en la siguiente tabla, en la que se indica la denominación del artista o grupo, número de suscriptores, número de vídeos y visualizaciones.

\begin{tabular}{|c|c|c|c|}
\hline Bandas & Suscriptores & Número de vídeos & Visualizaciones \\
\hline Extremoduro & 60.956 & 25 & 27.640 .585 \\
\hline Bebe & 34.146 & 23 & 10.857 .933 \\
\hline Robe & 13.576 & 10 & 4.408 .850 \\
\hline Los Ganglios & 4.580 & 83 & 2.018 .687 \\
\hline Divan du don & 811 & 49 & 271.553 \\
\hline Gecko turner & 0 & 6 & 111.404 \\
\hline Chloe Bird & 311 & 12 & 105.944 \\
\hline Javier Alcántara & 74 & 103 & 61.380 \\
\hline Pablo Guerrero & 154 & 2 & 45.848 \\
\hline Ruben Rubio & 162 & 24 & 45.723 \\
\hline Kaxta & 20 & 7 & 38.351 \\
\hline Marcos Bayón & 57 & 11 & 32.473 \\
\hline Acetre & 103 & 4 & 28.697 \\
\hline Sínkope & 0 & 13 & 27.893 \\
\hline Olivenza & 85 & 4 & 22.924 \\
\hline Cajón de sastre & 28 & 40 & 17.840 \\
\hline Inlavables & 20 & 42 & 15.872 \\
\hline Duende Josele & 0 & 14 & 15.418 \\
\hline $\begin{array}{l}\text { Los niños de los } \\
\text { ojos Rojos }\end{array}$ & 80 & 13 & 13.761 \\
\hline Hemingway & 47 & 10 & 12.709 \\
\hline Alé Canalla & 54 & 5 & 12.023 \\
\hline Luís Pastor & 35 & 310 & 10.176 \\
\hline Cira \& Ulises & 27 & 21 & 9.267 \\
\hline Omnia Transit & 42 & 15 & 7.818 \\
\hline Bucéfalo & 34 & 67 & 6.590 \\
\hline Da & 28 & 20 & 4.754 \\
\hline Supertennis & 26 & 8 & 4.070 \\
\hline $\begin{array}{l}\text { Rui Díaz y la } \\
\text { contrabanda }\end{array}$ & 44 & 5 & 3.807 \\
\hline Mili Vizcaíno & 45 & 1 & 3.495 \\
\hline Subterráneos & 32 & 17 & 3.119 \\
\hline
\end{tabular}

Tabla 1. Resultados de los canales de YouTube de las bandas extremeñas organizados de mayor a menor en cuanto número de visualizaciones Octubre/Noviembre 2015. 
Una vez realizado el análisis de los diferentes canales de las bandas extremeñas. Nos hemos encontrado con los siguientes datos:

En primer lugar, encontramos a la banda extremeña de rock por excelencia que comenzó su andadura en la mitad de los años ochenta. Extremoduro, con su líder Roberto Iniesta Ojeda originario de la ciudad cacereña de Plasencia aunque afincado en el País Vasco, cuentan con una cifra de 27.640 .585 visualizaciones, por lo tanto ocupando el primer puesto en el ranking. Su importante bagaje con más de diez discos editados y publicados es uno de los motivos que hacen que sea la primera banda. Observamos cómo cuentan con 25 vídeos subidos al canal en cuyo caso, en muchas ocasiones son canciones de sus discos sin ningún tipo de trabajo visual. Encontramos vídeos con solo el audio con una imagen de la portada del disco. No hay que olvidar que YouTube es un medio muy importante para a dar conocer las canciones de un disco ya que muchos usuarios utilizan YouTube para escuchar música. Por otro lado, encontramos la cifra de 60.956 suscriptores al canal. Sin duda una cifra muy importante. El hecho de contar con ese número de subscriptores hace que los contenidos que publica la banda en su canal son directamente consumidos por los usuarios suscritos sin tener que invertir en publicidad dentro de YouTube o realizar campañas para llegar a sus consumidores objetivos.

En YouTube es muy importante el número de suscriptores, ya que no funciona como una red social en el que un usuario puede pedir amistad para que le sigan o seguir a alguien para ser seguido. Sino que es el usuario el que por decisión propia decide seguir a un grupo, un realizador, un creativo en definitiva porque realmente le interesa su trabajo. Es decir, los subscriptores a canales pueden ser uno de los datos más importante para una banda.

En segundo lugar, encontramos a la artista extremeña Bebe con unos datos de 10.857.933 visualizaciones. Es una artista reconocida a nivel nacional e incluso en otros países como Francia, Turquía, Italia y gran parte de Sudamérica. En su canal hemos encontrado un total de 23 vídeos con un número de 34.146 suscriptores. Hemos podido observar que los contenidos actualizados en octubre de 2015 son normalmente 
actuaciones en directo conocidos como showcase que es una estrategia de hacer llegar de un modo más atractivo los nuevos contenidos audiovisuales a la gente. Además, Bebe recientemente ha publicado su nuevo disco "cambio de piel" por lo que gran parte de los contenidos subidos al canal de YouTube tienen una finalidad de marketing y venta del disco. Habría que analizar la potencialidad de venta y marketing de los archivos por medio de la red (Caldera y León, 2010).

En tercer lugar encontramos el proyecto en solitario de Robe junto a músicos extremeños como Álvaro rodríguez, Dave Lerhman, Lorenzo González, entre otros, que sin duda nos aporta unos datos muy importantes gracias al tirón mediático de Roberto Iniesta.

En cuarto lugar encontramos a los Ganglios, importante paradigma de este estudio. En esta banda originaria de Extremadura pero actualmente afincada en Barcelona, observamos unos datos muy positivos con 2.018.687 visualizaciones sin ningún tipo de apoyo de discográfica u otro medios de comunicación. Sin más que la gestión de sus contenidos a través de la red mediante redes sociales, YouTube, fan page de Facebook y otros medios. Encontramos una gran producción audiovisual en su canal con 83 vídeos en los que se mezclan vídeos de información sobre conciertos, publicidad de futuros espectáculos, videoclips de propia producción con una importante carga irónica y de humor que sin duda es la fórmula que les ha llevado a ser una de las bandas más reconocidas del panorama musical extremeño y a nivel nacional. Cuentan con un total de 4.580 suscripciones.

En quinto lugar encontramos a Diván du don con 271.553 visualizaciones, 49 vídeos y 811 suscriptores siendo interesante ya que llevan una autogestión de todos sus contenidos. Seguidos del pacense Gecko Turner con 111.404 visualizaciones y tan solo 6 vídeos. Hemos de decir que este último publica sus contenidos audiovisuales en canales de distribuidoras musicales que cuentan con una importante bolsa de suscriptores que hacen que los contenidos lleguen a un mayor número de usuarios y por tanto facilitando mucho el trabajo arduo que ha de llevar un artista si pretende arrancar buenas cifras con un canal propio de YouTube. 
En la zona media de la tabla encontramos a artistas como la reciente cantautora extremeña Chlóe Bird con 105.944 visualizaciones, bandas como Sínkope con 27.893, el famoso cantautor extremeño Luis Pastor o Pablo Guerrero.

Uno de los datos más interesantes es la parte inferior de la tabla en el que encontrarnos el panorama emergente de la música extremeña. Quizás es ahí donde se puede dar mayor relevancia al estudio. Observamos cómo las bandas nuevas como Ale canalla, Cira \& Ulises, Da, Rui Díaz entre otros cuentan con poco material audiovisual en relación con los artistas consagrados. Es por una cuestión de tiempo y también evidentemente de recursos económicos. Los mejores datos están relacionados en muchas ocasiones con la creación de contenidos audiovisuales con una calidad importante en cuanto a la realización audiovisual. Aquellos grupos que tienen más contenidos audiovisuales y de mayor calidad técnica hacen que sus contenidos tengan más números de visualizaciones. Pero también encontramos a bandas que llevan una larga trayectoria musical como Bucéfalo que en cambio están en una situación en la tabla muy baja. Por lo que el reconocimiento a una carrera musical no se ver reflejada en los datos que nos aporta YouTube.

Otro aspecto a destacar es que algunas bandas no necesitan mucho material audiovisual para tener buenos datos estadísticos. Por ejemplo, Pablo Guerrero con tan solo dos vídeos en su canal cuenta con 45.848 visualizaciones. Responde a que Guerrero es un cantautor con una importante trayectoria que hace que con solo dos vídeos aglutine un gran número de visualizaciones. Por ello, no depende de la cantidad de material audiovisual que se suba al canal, si no a cuestiones precedentes como la fama del artista, la calidad de los vídeos e interés de los contenidos que se suben a los diferentes canales. 


\begin{tabular}{|c|c|c|c|c|c|}
\hline Bandas & +2 años & 1 año & 6 meses & - 6 meses & Comentarios \\
\hline Extremoduro & 17 & 7 & - & - & 5.988 \\
\hline Bebe & 8 & - & - & 15 & 2.471 \\
\hline Robe & - & - & - & 10 & 1.668 \\
\hline Los Ganglios & 40 & 9 & 4 & 5 & 1.161 \\
\hline Divan du don & - & 4 & 19 & 27 & 45 \\
\hline Gecko turner & 3 & - & - & 2 & 5 \\
\hline Chloe Bird & 7 & 4 & - & - & 137 \\
\hline Javier Alcántara & 100 & - & 1 & 2 & 1 \\
\hline Pablo Guerrero & 2 & - & - & - & 33 \\
\hline Ruben Rubio & 22 & 12 & - & 2 & 68 \\
\hline Kaxta & 7 & - & - & - & 33 \\
\hline Marcos Bayón & 6 & 10 & - & 1 & 40 \\
\hline Acetre & 4 & - & - & - & 20 \\
\hline Sínkope & - & 1 & - & - & 19 \\
\hline Olivenza & 4 & - & - & - & 23 \\
\hline Cajón de sastre & 25 & 10 & 1 & 3 & 12 \\
\hline Inlavables & 21 & 18 & 1 & - & 11 \\
\hline Duende Josele & - & 4 & 2 & 10 & 35 \\
\hline $\begin{array}{l}\text { Los niños de los ojos } \\
\text { Rojos }\end{array}$ & - & - & - & 13 & 0 \\
\hline Hemingway & 8 & 2 & - & - & 12 \\
\hline Alé Canalla & - & 5 & - & - & 7 \\
\hline Luís Pastor & - & - & - & 310 & 50 \\
\hline Cira \& Ulises & 21 & - & - & - & 9 \\
\hline Omnia Transit & 7 & 5 & 1 & 2 & 9 \\
\hline Bucéfalo & - & 66 & - & 1 & 0 \\
\hline Da & 19 & 7 & - & 4 & 12 \\
\hline Supertennis & 1 & 6 & - & 2 & 1 \\
\hline $\begin{array}{l}\text { Rui Díaz y la } \\
\text { contrabanda }\end{array}$ & 4 & 1 & - & - & 1 \\
\hline Mili Vizcaíno & 2 & - & - & - & 4 \\
\hline Subterráneos & 3 & 11 & - & 3 & 6 \\
\hline
\end{tabular}

Tabla 2. Frecuencias de subidas de vídeos a los canales e interacción entre los usuarios y los videos mediante comentarios.

En cuanto a la interacción de los usuarios con los contenidos de los canales de YouTube observamos cómo en los primeros puestos coinciden con que existen unos importantes datos de comentarios. Es decir bandas como Extremoduro, Bebe, Robe o los Ganglios son los que más comentarios tienen en sus vídeos.

Y así va decreciendo hasta los puestos más inferiores. Vemos como las bandas con más 
éxitos motivan mayor interacción de los usuarios con los vídeos mediante comentarios y además como suelen coincidir que son aquellos vídeos que corresponden a lanzamientos de nuevos discos o de temas que son muy conocidos. También, observamos como en ocasiones existen interacciones entre usuarios que opinan sobre los temas, las letras, la música, etc.

Por otro lado, encontramos que los mejores datos son de aquellas bandas que mantienen una continuidad en la publicación de vídeos. Bandas como Extremoduro no tienen una continuidad en sus publicaciones, hace más de un año que no publican nada y tienen muy buenos datos. Este hecho se explica por su reconocimiento artístico por medios de comunicación, crítica y público haciendo que sus datos sean positivos aunque no estén actualizados con mucha asiduidad.

Sin embargo, vemos que bandas como los Ganglios tienen una importante continuidad desde hace más de cinco años, publicando un gran número de contenidos audiovisuales. Al igual que Diván du Don con una progresiva subida de vídeos que mantienen el interés de los usuarios.

Hemos observado también como algunos canales están llenos de contenidos que nada tienen que ver con lo musical, siendo una mala imagen para las bandas y artistas. En ocasiones se mezclan sus propios contenidos como banda con vídeos de sus intereses. Este hecho hace que los canales no tengan una homogeneidad y haga que el usuario no se interese por suscribirse a dicho canal.

\section{Conclusiones}

Analizando el panorama musical extremeño a través de los canales de YouTube, encontramos como se ve recogido un importante archivo audiovisual de toda la producción musical popular de nuestra comunidad en esta plataforma. Por ello, supone una importante fuente documental para recoger y analizar información.

El trabajo ha puesto de relieve la realidad en la que se encuentra mucho de los 
contenidos de las bandas y autores musicales de nuestra comunidad. No existiendo ningún tipo de control documental sobre estos contenidos audiovisuales tal y como señalan algunos autores (Cadera y Arranz, 2012) (ILópez, 2007) (Hidalgo, 2013) (Giménez, 2012).

Seguramente como región debiera controlarse la información audiovisual en la red a nivel de la producción televisiva (Blanco y Póveda, 2015) controlando información que actualmente está deposita en servidores de información en muchos casos con falta de consistencia e integridad documental.

Existen algunas plataformas digitales como es cantarranas (http://cantarranacorps.blogspot.com.es/), quienes de un modo altruista y sin ningún tipo de apoyo económico se encargan de hacer un inventario muy importante de todo el material que se genera en nuestra comunidad autónoma en cuanto a lo musical. Organizándose por géneros, años de producción, tipo de contenidos, formatos, estilos musicales, onomásticamente, etc. E incluso tienen un inventario de todos los conciertos que se han llevado a cabo en Extremadura desde los años ochenta hasta la actualidad.

Quizás, sea un trabajo que puede nacer de un amante de la música como puede ser quien gestione cantarrana. Pero sin duda, debían ser los organismos públicos quienes preserven nuestra cultura y nuestra memoria que en ocasiones está desparramada por la red. Sería, interesante llevar a cabo la creación de canales de YouTube o listas de reproducción de los vídeos extremeños con el fin de que exista una organización dentro de la plataforma y además de mayor visibilidad a la música producida en nuestra comunidad.

Por otro lado, hemos llegado a la conclusión de que un mayor número de contenidos audiovisuales no mejoran las cifras de visualizaciones. En muchas ocasiones los grandes datos están relacionados con la popularidad de los autores.

Además, hemos observado como una buena gestión de los canales de YouTube hacen que los grupos tengan mayor visibilidad en la plataforma. Durante el estudio hemos 
observado como algunos grupos contaban con vídeos en diferentes canales. En muchas ocasiones, a causa de que se han publicado en diferentes canales con diferentes direcciones url. Esto hace que el usuario tenga más dificultades para recuperar contenidos de las bandas.

Algunas bandas, tienen sus contenidos descentralizados porque son publicados por sus productoras haciendo que tengan mayor visualización ese vídeo pero menor visualización el resto.

Otro de los errores que hemos encontrado en muchos de los vídeos de los canales es que un gran número de vídeos están mal nombrados, siendo muy difícil su recuperación. Además, las etiquetas no están aplicadas haciendo que los vídeos tengan menor visibilidad. También, las descripciones de los vídeos son casi inexistentes. Sería interesante introducir una serie de datos, como el realizador, fecha de cuando fue grabado, reparto, pieza musical, etc.

\section{Referencias bibliográficas}

ANTOLIN PRIETO, Rebeca (2012). YouTube como paradigma del vídeo y la televisión en la Web 2.0. Madrid: Universidad Complutense (Tesis doctoral) http://eprints.ucm.es/16111/1/T33817.pdf [Consultado: 31 de enero de 2015]

BLANCO-IZQUIERDO, María Jesús; PÓVEDA-LÓPEZ, Inés Carmen (2015). Metadatos Documentales en el Canal Extremadura Televisión // Cuadernos de Documentación $\begin{array}{llll}\text { Multimedia, } & \text { vol. 26, 113-132. }\end{array}$ https://revistas.ucm.es/index.php/CDMU/article/view/50633/47035 [Consultado: 31 de enero de 2015]

CALDERA-SERRANO, Jorge; ARRANZ-ESCACHA, Pilar (2012). Documentación audiovisual en televisión. Colección El profesional de la información; 13. Barcelona: Editorial UOC. 
CALDERA-SERRANO, Jorge; LEÓN-MORENO, José-Antonio (2010). Marketing analysis of the web-based commercialization of television audiovisual files: opportunities and implications // Investigación bibliotecológica, vol. 24, núm. 52, pp. 217-235.

CALDERA-SERRANO, Jorge (2006). Terminological control of "anonymous groups" for catalogues of audiovisual television documents // Journal of librarianship and information science, vol. 38, núm. 3, pp. 187-195.

GIMENEZ-RAYO, Mabel (2012). La documentación audiovisual en televisión en el mundo 2.0: retos y oportunidades. $\quad / / \quad$ Trípodos. $31 \quad$ (2012) 79-97. http://tripodos.com/index.php/Facultat_Comunicacio_Blanquerna/article/download/39/ 24 [Consultado: 31 de enero de 2015]

HERRERA, Raquel; CODINA, Lluís (2015). Redes Sociales Visuales: Caracterización, Componentes y posibilidades para el SEO de Sitios Intensivos en Contenidos. Cuadernos de Documentación Multimedia, vol. 26, pp. https://revistas.ucm.es/index.php/CDMU/article/view/50627/47029 [Consultado: 31 de enero de 2015]

HIDALGO GOYANES, Paloma (2013). Patrimonio audiovisual en televisión. // Marcos Recio, Juan Carlos (coords.). Gestión del patrimonio audiovisual en medios de comunicación. Colección Ciencias de la información. Comunicación audiovisual; 18. Madrid: Síntesis, 2013. 53-82.

LÓPEZ-DE-QUINTANA, Eugenio (2007). Transición y tendencias de la documentación en televisión: digitalización y nuevo mercado audiovisual. // El profesional de la información.16:5 (julio-agosto 397-408. http://www.elprofesionaldelainformacion.com/contenidos/2007/septiembre/01.pdf. [Consultado: 31 de enero de 2015].

PÓVEDA-LÓPEZ, IC; CALDERA-SERRANO, J; POLO-CARRION, JA (2010). Definición del objeto de trabajo y conceptualización de los Sistemas de Información Audiovisual en 
Televisión // Investigación bibliotecológica, vol. 24, núm. 50, pp. 15-34.

REGUILLO, Rosanna (2012). Navegaciones errantes. De músicas, jóvenes y redes: de Facebook a Youtube y viceversa.

Nueva Época, nº 18, (Julio-Diciembre 2012) 135-171

http://148.202.18.157/sitios/publicacionesite/pperiod/comsoc/revista18/6.pdf

SIRI, Laura (2008). Un análisis de YouTube como artefacto sociotécnico. // Diálogos de la Comunicación, núm. 77. http://www.dialogosfelafacs.net/ [Consultado: 31 de enero de 2015]. 